\title{
EL PROCESAMIENTO COGNOSCITIVO DEL DISCURSO LITERARIO
}

\section{Introducción. Objetivos. Problemas}

En esta ponencia nos proponemos aplicar algunos de los resultados recientes de la psicología cognoscitiva al estudio del discurso literario. Estos resultados pertenecen a los procesos de lectura y comprensión del discurso, así como a las maneras en que la información textual se almacena y se rescata de la memoria.

En el estudio de la literatura, tales resultados son de gran importancia para el análisis de los llamados procesos de "interpretación" y de "recepción". Desde luego, el problema de "cómo entendemos" un texto literario siempre ha sido una preocupación fundamental tanto de'l estudio literario tradicional como del más reciente: en hermenéutica y en semántica se ha intentado dar cuenta de los distintos tipos de significado de los textos literarios. Tal significado se detemina en un proceso de interpretación. La interpretación puede ser "formal", es decir formuláda en términos de una semántica explícita (gramatical o lógica), o bien puede ser más subjetiva en el sentido de que un oyente/lector le asigne un significado a un discurso.

Con el fin de profundizar más en las maneras en que los lectores reales de literatura entienden o interpretan un texto literario - Io que es una condición necesaria para un procesamiento ulterior, como por ejempio la asignación de valores al texto- se necesitan datos empíricos de la psicología acerca de cómo los usuarios de la lengua leen y comprenden un discurso.

La aplicación de la psicología al estudio de la literatura se da dentro de un desarrollo general de la poética hacia lo interdisciplinario. Nos damos cuenta, cada vez más, de que la literatura no sólo es un conjunto particular de discursos, 
posiblemente definidos sobre la base de propiedades textuales específicas, sino que, al mismo tiempo, la literatura debería considerarse en términos de los distintos aspectos de la comunicación. Así, los textos literarios pueden tomarse, en la pragmática, como un tipo de actos de habla rituales; intentaremos mostrar cuáles son Jas condiciones, funciones $y$ efectos sociales de tales actos en el contexto comunicativo de escritores, editores, lectores, críticos, maestros, etc. En otras palabras, ya no intentaremos definir la "literariedad" sólo en términos de las estructuras del discurso literario, sino más bien en términos del papel que desempeñan tales discursos en los procesos de interacción sociocultural. Un aspecto básico de estos procesos comunicativos son las distintas operaciones cognoscitivas de los distintos participantes, ya mencionados, del "contexto literario". Para poder explicar cuáles son las funciones y los efectos particulares de la literatura, debemos saber cómo los lectores entienden, valoran, memorizan, parafrasean, resumen, reproducen (entre otras cosas) los textos literarios. Lo mismo es necesario para la explicación de los procesos de producción: qué condiciones sociales de los escritores y qué "conjunto cognoscitivo" personal (conocimientos, opiniones, actitudes, sentimientos, etc.) operan en los procesos cognoscitivos implicados en la producción de un texto literario.

En esta ponencia sólo podemos intentar un primer paso hacia el análisis cognoscitivo de los procesos de comunicación literaria. En primer lugar, no trataremos aquí los problemas específicos implicados en la producción de un texto literario, sino que prestaremos mayor atención a la recepción: percepción, lectura, comprensión/interpretación, memorización, etc. Además, limitaremos aún más esta presentación a los procesos de comprensión y de memorización, por lo que tendremos que descuidar los aspectos emotivos, valorativos y de actitud implicados en la recepción literaria. Hemos ya sugerido que, ante todo, debe saberse algo acerca de la comprensión antes de hablar de la valoración. Prestaremos atención, muy brevemente, a la relación entre estos aspectos importantes de la comunicación literaria. Por último, es imposible tratar en detalle todos los procesos cog- 
noscitivos que son relevantes en la lectura y la comprensión de un texto (literario); de manera que prestaremos atención especial a los aspectos semánticos de los procesos de comprensión literaria. Esta es, precisamente, el área tradicionalmente llamada de "interpretación literaria". Consideramos que un análisis cognoscitivo puede aclarar, o explicitar, los distintos problemas de este tipo de interpretación literaria. Así, un problema que debemos tratar de resolver es el que se refiere a las oraciones muy ambiguas, vagas $o$ incompletas, que pueden ocurrir en cierto tipo de textos literarios: ¿Cómo las entienden realmente los lectores? En lugar de un acercamiento normativo ("lo que los lectores deberian interpretar"), proponemos un acercamiento empirico ("lo que los lectores realmente hacen cuando Ieen literatura").

\section{Procesos COgnoscitivos EN LA COMPRENSIÓN DEL DISCURSO}

Durante los últimos años se ha prestado más atención a los procesos cognoscitivos implicados en la comprensión del discurso. Mientras la psicolingüística y la psicología experimental originalmente se interesaban más en el procesamiento cognoscitivo de palabras, frases y oraciones, primero desde un punto de vista sintáctico y después semántico, ahora presenciamos la extensión de esta primera investigación hacia unidades de información más complejas. Los psicólogos y estudiosos que trabajan en el campo de la llamada "inteligencia artificial" se han interesado, por ejemplo, en las maneras en que un cuento se comprende y se representa en la memoria. En esta sección resumiremos brevemente los puntos principales de esta postura. Para presentar los detalles debemos remitirnos a los numerosos libros $y$ artículos sobre este tema que han aparecido en los últimos años. Nuestra presentación se dará, en parte, dentro de la perspectiva de nuestro propio trabajo teórico y experimental sobre las estructuras del discurso y el procesamiento cognoscitivo del mismo.

Un primer aspecto básico de la comprensión que ha aten- 
dido la psicología del procesamiento del discurso - y de la información compleja en general- es la naturaleza predominantemente semántica de los procesos implicados. La comprensión de un texto, requiere fundamentalmente, que un usuario de la lengua, -es decir, un oyente o lectorasigne una estructura semántica a las unidades respectivas del texto. Así, gradualmente construye una representación conceptual o semántica del texto en la memoria. La información "estructural superficial", por ejemplo la morfofonológica y la sintáctica, del texto "se traduce" o "se transforma" en significados que se representan cognoscitivamente en términos de "conceptos".

Este proceso cognoscitivo de comprensión conceptual se da en varias fases subsecuentes y en varios niveles. La función de la memoria es crucial en las distintas fases de la comprensión del discurso. En general, hacemos una distinción teórica entre la memoria a corto plazo (STM) y la memoria a largo plazo (LTM). La memoria a corto plazo, que tiene una capacidad limitada, es el Iugar en el que se analiza e interpreta toda la información que entra a través de nuestros distintos sentidos. En la comprensión del discurso esto significa que, en la memoria a corto plazo analizamos secuencias de sonido como fonemas, morfemas y estructuras sintácticas (de una lengua determinada) a las que asignamos significados conceptuales. La diferencia de la interpretación, tal como se especifica en la gramática, es que en la comprensión real los distintos procesos de análisis e interpretación pueden darse en un orden que varía estratégicamente. Así, nuestro conocimiento o nuestras expectativas acerca del significado de una palabra, frase u oración puede determinar su análisis sintáctico. La 'memoria a corto plazo aparentemente funciona como la llamada memoria trabajadora: percepción, comprensión, pensamiento, etc., se realizan allí. Sin embargo, la memoria a largo plazo es la bodega en que se deposita, con el tiempo, la información de la memoria a corto plazo. Aunque mucha información se almacena de esta manera en la LTM, esto no significa que en realiclad recordemos toda esa información, porque el recuerdo o el reconocimiento de la información depende de 
procesos de recuperación. De hecho, la mayor parte de la información que se guarda en la LTM, después de su procesamiento en la STM, nunca puede recuperarse. Un ejemplo importante de información que se "olvida" de esta manera son las distintas estructuras de superficie de un texto. Casi nunca podemos recordar la estructura exacta, palabra por palabra, de las oraciones o los textos que hemos oido o leído. Aun después de varias oraciones ya no podemos recordar una oración que hayamos leído antes. De hecho, esto ni siquiera es necesario: en cuanto hemos comprendido tal oración, es decir que le hemos asignado un significado conceptual, ya no necesitamos la información de la estructura superficial. Veremos en la siguiente sección cómo esta caracteristica fundamental de la comprensión de la lengua puede no resultar cierta en algunos tipos de comunicación literaria.

Hemos visto que a un texto se le asigna gradualmente un. significado conceptual en la STM. Dado que la STM tiene una capacidad limitada para conservar, este proceso debe darse por "paquetes". No "leemos por completo" una oración, ni menos aún un texto, y les asignamos un significado en la STM. La interpretación cognoscitiva (comprensión) comienza de inmediato, y en la medida en que la capacidad de conservación de la STM lo va necesitando, la información conceptual resultante se transporta a la LTM. En otras palabras, si suponemos que una oración se interpreta como una secuencia de proposiciones, debemos tomar en consideración que tal secuencia se guarda en la LTM en cuanto deban interpretarse oraciones subsiguientes.

Sin embargo, lo típico para la comprensión del discurso es el requisito general de que los textos deben tener una coherencia. Esto significa, entre otras cosas, que las oraciones respectivas de un texto deberían estar (semánticamente) conectadas. Para hacer esto en la STM, debemos asumir que una oración anterior, o más bien sus proposiciones subyacentes, todavia están disponibles en la bodega ("amortiguador") de la STM, para que puedan relacionarse con las proposiciones de la oración que se interpreta. Todo este proceso es ciclico: apenas se incluye una nueva información 
en la STM amortiguador, y apenas la información vieja ya no es necesaria para el establecimientorinmediato de la coherencia, por lo menos una parte dểa viejà información se almacenará en la LT.M. De allí, puede regresarse cuando sea necesario (si se almacena de tal manera que la información sea recuperable, desde luego). La información de la oración anterior debe permanecer en la STM, como dijimos, para establecer la coherencia semántica. Esto implica, por ejemplo, algunas relaciones condicionales entre las proposiciones (es decir, causas, razones y consecuencias). De la misma manera, podemos querer "tener presente" que el (los) participante (s) de un hecho denotado por la oración anterior también aparece en el hecho denotado por la oración siguiente. Si no fuera éste el caso, no podríamos interpretar rápidamente, por ejemplo, los pronombres.

El tipo de interpretación semántica del discurso del que hablamos aquí puede llamarse local: implica la interpretación de oraciones y el establecimiento de relaciones de coherencia entre oraciones sucesivas. Sin embargo, en la comprensión del discurso también se da un proceso de interpretación global. Tal interpretación global es necesaria para que el lector pueda establecer el lema, el asunto o la esencia de un texto o un fragmento de texto. Es decir que cuando está leyendo y comprendiendo una secuencia de oraciones en un texto, el lector sabrá, o intentará saber, de qué se trata la secuencia en su tótalidad. Este tipo de interpretación global se explicita en términos de macroestructuras semánticas. Tales macroestructuras también son secuencias de proposiciones, pero en otro nivel de interpretación. Así, podemos representar, en un nivel global, el contenido semántico de un cuento acerca del viaje de John a México mediante una (macro) proposición: "John hizo un viaje a México". Somos capaces de hacer esto a través de una serie de operaciones semánticas específicas, que se llaman macrorreglas. Estas macrorreglas organizan y reducen la información compleja de las proposiciones de un texto. Nos permiten reducir miles de proposiciones a unas pocas (macro) proposiciones. Las macroproposiciones representan los "mismos" hechos, pero en un nivel más "distante" o más 
"abstracto". Las distintas macrorreglas funcionan de la siguiente manera: a partir de una secuencia de proposiciones, en primer lugar, suprimen las proposiciones que son (consideradas por el lector) irrelevantes para la interpretación del resto del texto; en segundo lugar, intentarán generalizar secuencias de proposiciones en términos de una proposición más general (con la ayuda de un "superconcepto", por ejemplo "mascotas" en lugar de "perro", "gato", "canario", etc.); en tercer lugar, una macrorregla intentará juntar información proposicional que represente los distintos aspectos o sucesos de un episodio socialmente conocido, y luego substituir las distintas proposiciones con una proposición que represente el episodio en su totalidad, como por ejemplo "John hizo un viaje a México" para todas las proposiciones que describen las distintas acciones de ese viaje. Esta última regla se liama la regla de construcción. Sólo es posible aplicar esta regla cuando se tiene cierto conocimiento del mundo: debemos saber cómo tomar un avión, llegar al aeropuerto, etc., porque si no tenemos estos conocimientos acerca de los episodios sociales, ni siquiera podemos saber cuál es el episodio global representado, por lo que no podremos construir, una macroproposición.

En el procesamiento cognoscitivo, la formación de macroproposiciones, mediante la aplicación de diversas macrorreglas, se da en la STM al mismo tiempo que interpretamos las oraciones respectivas de un texto. Es decir que, con frecuencia, podemos conectar localmente esas oraciones sólo si sabemos cuál es el asunto global del texto o del fragmento. Ahora vemos que en el proceso cíclico de la comprensión local del discurso, deberíamos asumir que el amortiguador de la STM no sólo debería contener información de la oración anterior, sino también la macroproposición "válida" para este fragmento del texto.

Incliso deberiamos asumir que, dado que las macroproposiciones sólo pueden formarse sobre la base de nuestro conocimienlo del mundo, la STM también contiene, por lo menos momentáneamente, proposiciones que vienen de ese conocimiento del mundo que está almacenado en la LTM. De hecho, aun para la conexión local de las oracio- 
nes, con frecuencia necesitamos un conocimiento del mundo para establecer la coherencia: cuando leemos John mostró su bolelo a la señorita del mostrador, nuestro conocimiento acerca de tomar aviones debe proporcionarnos la información de que en un aeropuerto hay mostradores, de que en general tenemos un boleto para este tipo de transporte, y de que debemos mostrar ese boleto a la persona que atiende el mostrador, etc. El hecho de que este tipo de conocimiento está presente cognoscitivamente también se señala en lo gramatical por los artículos definidos de esta oración. El conocimiento del mundo que tenemos en la LTM es tan voluminoso que para que lo utilicemos de manera efectiva, debemos suponer que está muy bien organizado. Uno de los principios de organización que acabamos de presenciar es el de los marcos convencionales, o sea una serie de proposiciones sobre algún episodio social, tal como tomar un avión o comer en un restaurante. Tal marco cognoscitivo ros permite participar efectivamente en esos episodios, interpretar correctamente los sucesos y acciones de esos episodios, derivar las expectativas necesarias sobre lo que puede suceder o sucederá, $y$ por último comprender discursos acerca de tales episodios. Asi, un texto es comprensible para nosotros, en último análisis, si entendemos cuáles hechos denotados por el texto "pueden juntarse", según nuestro conocimiento del mundo. De la misma manera, tenemos conocimientos organizados acerca de la estructura de sillas, cuartos, caballos, pueblos y caras. En otras palabras, todos los procesos cognoscitivos, incluso la comprensión del discurso, se basan en nuestro sistema de conocimientos almacenado en orden en la L.TM.

Además de la interpretación semántica local y global de secuencias oracionales textuales, el lector por último tratará también de asignar las llamadas superestructuras esquemáticas. Un ejemplo típico de tales estructuras esquemáticas son las estructuras narratitjas, expresaclas en relatos. Nótese que esas estructuras, que consisten de una secuencia jerairquica de categorías esquemáticas (por ejemplo ambiente, complicación y resolución, etc. en un relato), son independientes de la estructura semántica del texto: un cuento pue- 
de tratar casi cualquier tema y seguir siendo un cuento. Sólo hay algunos límites semánticos para las diversas categorías esquemáticas. Tales límites funcionan en el nivel global de las macroestructuras: por ejempio, una resolución puede requerir que un fragmento como totalidad, o sea una macroproposición, denote una acción específica de un ser humano. Otras estructuras esquemáticas son las de una argumentación o de un estudio psicológico. El ejemplo clásico en literatura es la segmentación de tragedias en distintos "actos", que más o menos corresponden a las categorías narrativas básicas. Así, en el proceso de lectura y comprensión, el lector intentará utilizar el esquema (por ejemplo narrativo), con el fin de organizar la macroestructura del texto. Las macroproposiciones pueden caber en las categorías de un esquema convencional.

Con este último ejemplo, se puede advertir una vez más que la lectura y la comprensión, en distintos niveles, básicamente implican la asignación de distintas estructuras. Este es un aspecto fundamental de cualquier tipo de procesamiento de información. En cuanto podemos estructurar la información, reducimos la dificultad de procesarla: organizamos la información en "paquetes", y estos paquetes pueden ligarse a otros paquetes de información en la memoria. Por lo tanto, es más fácil leer, comprender y memorizar una secuencia de palabras cuando tiene una estructura sintáctica oracional. Lo mismo sucede cuando organizamos secuencias de oraciones por relaciones de coherencia en el nivel local $y$ en el global. $\mathrm{X}$ por último, organizamos la (s) macroestructura (s), es decir los temas o asuntos respectivos del texto, no sólo mediante las ligas usuales de coherencia lineal, sino también según una superestructura esquemática. Dado que esta superestructura esquemática convencionalmente es conocida por los usuarios de la lengua de una comunidad sociocultural determinada, es fácil manejar tales esquemas en la producción y la comprensión del discurso.

$\mathrm{El}$ principio fundamentaI que caracteriza la siguiente fase de la comprensión del discurso, a saber el almacenamiento de información textual en la memoria (a largo plazo), es que este almacenamiento se da en términos de las estructu- 
ras asignadas al texto durante la comprensión (en la STM). De esta manera, cada parte de información, o sea cada proposición, tiene su propio valor estructural, que puede medirse en términos del número de relaciones estructurales que tiene con otras proposiciones, con macroproposiciones o con estructuras esquemáticas. Desde luego, tales \igas entre proposiciones no sólo funcionan para la información del texto mismo, sino también para la información que ya estaba almacenada en la memoria o que proviene de una interpretación del contexlo comunicativo. Por eso, cuando leemos que John hace un viaje a México, la información recogida del texto también está ligada, como hemos visto, a nuestro conocimiento general sobre viajes en avión, así como a nuestro conocimiento sobre México y sobre John, y nuestros intereses, opiniones, actitudies, etc., ante este tipo de información. El estado cognoscitivo del lector, en el que todos estos factores desempeñan una función en la comprensión y el almacenamiento de la información que entra en un momento determinado, se llamará el conjunto cognoscitivo del usuario de la lengua. Este conjunto cognoscitivo es variable en lo que se refiere al contexto: en otrá situación el lector puede tener distintos conocimientos, opiniones, necesidades, actitudes, etc., y esto afectará las maneras en que se comprende la información y se liga a otra información en la memoria. De la misma manera, distintos lectores tendrán un conjunto cognoscitivo distinto. Esto explica el que haya variaciones en los modos en que los lectores comprenden y aimacenan el mismo texto, aunque en la interacción comunicativa, descle luego, haya una identidad convencional mínima entre estas interpretaciones; si no fuera así, la comunicación sería imposible. Por lo tanto, de acuerdo con su conjunto cognoscitivo, los lectores pueden también construir diferentes macroestructuras, es decir asignar diferentes temas o asuntos a un texto. Lo que es importante o relevante en un texto para un lector puede serlo menos para otros.

La representación de un texto en la memoria, pues, es una estructura conceptual compleja, que está organizada tanto interna como externamente por distintas ligas es- 
tructurales. Debe subrayarse que la representación final es el resuitado de varias transformaciones. El primer conjunto de transformaciones ya ha sido mencionado, a saber, las macrooperaciones ( macrorreglas), que suprimen, generalizan y construyen proposiciones en diversos macroniveles. Pero, debiclo a nuestros conocimientos generales y a otros factores de nuestro conjunto cognoscitivo, podemos aplicar todo tipo de supresiones, permutaciones, adiciones y substituciones. Así, podemos agregar a la representación del texto todo tipo de información que ya teníamos acerca de un episodio o de los objetos denotados por el texto.

Si consideramos ahora la fase final del procesamiento del discurso, a saber la recuperación y la reproducción o el uso cle la información en la memoria, nos damos cuenta cle que, en realidad, la organización estructural de la representación del texto es absolutamente crucial. El principio general es que la información que tenga mayor valor estructural será recuperada con más facilidad de la memoria que la información con menor valor estructural. Todos sabemos, por ejempio, que cuando leemos un texto, en general no podremos recuperar todas las oraciones o proposiciones del texto. Con frecuencia recordaremos lo importante o relevante (para nosotros), y sólo ocasionalmente recordaremos. los detalles. Después de algún tiempo será aún más difícíil recuperar incluso esos detalles. Se ha demostrado en diversos experimentos que especialmente las macroproposiciones, que representan la información "más importante" o "temática" de un texto, son las que pueden recuperarse mejor $y$ durante más tiempo. Ahora podemos explicar esto por el hecho de que toda macroproposición estará en un nivel más aito en la estructura jerárquica de la representación del texto; esto implica que la macroproposición tiene un alto valor estructural debido a sus múltiples ligas con: (i) las microproposiciones de las que se deriva, (ii) otras macroproposiciones, y (iii) las categorías esquemáticas (como por ejemplo, las de la estructura narrativa). Por el contrario, los cletalies sólo pueden estar ligados a una proposición, anterior o posterior. Sin embargo, si un "detalle estructural" está ligado con mucha de la información de nuestro "con- 
junto cognoscitivo, tal como una tarea, un interés, actitud valor, etc., este detalle puede "sobresalir" y recuperarse fácilmente.

Si se pide a los sujetos de un experimento de memoria que reproduzcan un texto que hayan leído, en primer lugar producirán las macroproposiciones esquemáticamente organizadas del texto, así como algunos "detalles sobresalientes". Por ejemplo, de las doscientas proposiciones originales de un cuento determinado, de inmediato recordarán sólo entre la mitad y una cuarta parte de ellas, inciuyendo la mayoría de las macroproposiciones y todo tipo de proposiciones transformadas (de otra manera). Después de algunos meses, sólo recordarán las macroproposiciones y habrán olvidado la mayor parte de los detalles. Sin embargo, después de largos lapsos, incluso las macroestructuras se desintegrarán.

Tenemos ahora una idea general de algunos de los procesos fundamentales y de los principios de la comprensión "normal" del discurso. Veamos ahora cómo sucede todo esto en la comprensión del discurso literario.

\section{Comprensión del discurso literario}

Los procesos y los principios de la comprensión del discurso presentados en la sección anterior son de carácter general. Es decir que caracterizan cualquier tipo de procesamiento de información semántica compleja. Esto significa que, en principio, también se aplican a la comprensión del discurso literario. En otras palabras, nuestros mecanismos cognoscitivos simplemente no nos permitirán comprender un discurso o una información de una manera diferente en lo fundamental. A este respecto, debe señalarse que el discurso Viterario y la comunicación literaria en general se ajustan a los principios de todo tipo de discurso y comunicación; por lo tanto, negamos estrictamente la naturaleza "específica" por completo de la llamada "interpretación literaria", según se postula normativamente en los estudios literarios tradicionales.

Este principio general del análisis de los procesos de com- 
prensión del discurso literario no implica que no tendría sentido buscar ciertos aspectos particulares de la manera en que los procedimientos generales se aplican o se usan en la interpretación del discurso literario. Esto no se debe, por lo menos en primer lugar, a las posibles estructuras específicas de los textos literarios, sino más bien a las funciones pragmáticas y socioculturales de la literatura en la interacción comunicativa. Es decir que muchos tipos de literatura, por ejemplo las novelas, no muestran estructuras que requerirían diferentes procesos semánticos de comprensión. Mientras que algunos textos literarios, como por ejemplo algunas formas de la poesía moderna, que sí parecen tener tales estructuras, pueden compartir esas estructuras con todo tipo de textos no literarios, como la publicidad y la conversación cotidiana. Lo que es común a ambos tipos de discurso es que el lector u oyente tratará de "darle sentido". Sin embargo, es diferente el contexto sociocultural y pragmático: el poema se produce, se lee y se entiende como un acto de habla que no necesariamente tiene las funciones pragmáticas "prácticas" usuales, como por ejemplo una afirmación (real), una pregunta, amenaza o promesa en nuestra conversación cotidiana, sino sólo o ante todo una función ritual. En este respecto el poema, tanto como la novela, pero también como el chiste y otros tipos de juegos de palabras, relatos, etc., de la comunicación no literaria, funcionan en un contexto en el que el hablante-escritor intentó cambiar, en primer lugar, el conjunto valorativo del lector respecto del texto mismo (o sus distintas características). Desde luego, esto no significa que el texto literario no funcione indirectamente como otro tipo de acto de habla, como por ejemplo, de hecho, una afirmación, una amenaza, una promesa, una felicitación, una pregunta, una petición o una protesta. Por último, esta función pragmática especifica de la literatura como un tipo de acto de habla ritual se especifica aún más por el contexto sociocultural, definido por los participantes específicos y sus diversos papeles o funciones (escritores, lectores, críticos, maestros, historiógrafos, editores, vendedores, etc.) implicados en los procesos de comunicación literaria, así como las diversas instituciones, acciones y conven- 
ciones que caracterizan los distintos marcos sociales en que se utiliza la literatura (el salón de clases, la conversación literaria, el periódico, el libro de texto, etc.). Es este fondo sociocultural el que establece en cada cultura cuáles discursos cuentan como, o se aceptan como, rituales o, más particularmente "iterarios". Ese mismo fondo determina nuestra conducla social en nuestra "interacción" con la liceratura: la manera en que nos interesamos en ella, la evaluamos, la compramos, la leemos, la comprendemos y hablamos de ella (y de nuestra comprensión de ellia).

Para este estudio, esto significa que el contexto sociocultural específico en el que funciona nuestro tipo de literatura puede requerir modos específicos de lectura, comprensión y uso del discurso. Una de esas convenciones ya ha sido mencionada: en la comunicación literaria el discurso no tiene, o no debería tener, un carácter primariamente instrumental, como sí lo tiene en el marco de los actos de habla cuyo propósito básico se define en términos de cambios en los conocimientos, deseos y acciones de los oyentes/ lectores, respecto de hechos extratexluales: un estado del mundo, una acción, una opinión del hablante, etc. Esto lleva al adagio intuitivo conocido de que en la comunicación literaria ( $y$ en la comunicación ritual en general) la atencion, o el enfoque de interpretación, del lector está "en el texto mismo". Debemos ahora tratar de explicitar más este principio, mediante la investigación de su base cognoscitiva.

En primer lugar, esta base cognoscitiva de la comunicación literaria pertenece a los contextos pragmático y sociocultural: un lector sabe cuáles son los marcos, convenciones y acciones que caracterizan típicamente la comunicación literaria. Así, además de la interpretación del texto, el iector estará obligado a interpretar la situación social, con lo que construirá el contexto específico (pragmático) requerido para una adecuada interacción literaria (ritual). Las indicaciones utilizadas en este proceso de interpretación pragmática vienen de varias fuentes: (sub) títulos del libro o discurso seleccionado para la lectura, conocimiento acerca de escritos y editores "literarios", el marco del contexto social 
especifico (casa, escuela) implicado, etc. Sobre la base de esta información, el lector sabe que posiblemente sea ritual el tipo de acto de habla que se realizará. Por lo tanto, esperará aigunos rasgos específicos del discurso y del escritor: espera que el escritor no tenga como propósito principal informarle acerca del "mundo" como es, ni que tenga propósitos específicos en lo que se refiere a los deseos, opiniones o acciones del oyente relacionados con este específico conocimiento del mundo. En otras palabras, los límites contextuales de la comunicación ritual inducen un conjunto cognoscitivo particular en el lector. Ese conjunto cognoscitivo determinará las maneras en que se analiza y se interpreta el texto. Uno de los factores del conjunto cognoscitivo, por ejemplo, es la tarea o el problema específico del lector al leer un texto. Así, en general el lector tiene la tarea de detectar cierto tema. Pero puede ser también que tenga que prestar atención especial a ciertas palabras, construcciones gramaticales u ortografía, como es el caso de un estudiante de gramática o de estilo, un corrector o el sujeto de un experimento psicológico. Este tipo de tarea puede liamarse no normal, porque no se centra en los temas globales semánticos y pragmáticos (macroestructuras) del discurso, como en el caso de la conversación cotidiana y del discurso impreso en periódicos, manuales, etc., en que la información se utiliza para una mayor (inter) acción. En otras palabras, en ciertos tipos de comunicación nuestro conjunto cognoscitivo puede inducir modos no normales de análisis e interpretación. Por ejemplo, esto puede implicar un tipo de mecanismo de selección, que en la STM elige la información específica requerida por el esquema del conjunto cognoscitivo, definido por una tarea particular. De tal manera, es posible seleccionar no sólo la información semántica, sino también la información de la estructura superficial de diversos tipos, por ejemplo ciertas estructuras fonológicas (rima, aliteración, esquema métrico, etc.) u operaciones sintácticas de naturaleza retórica o estilística 'paralelismo, inversión, semigramaticalidad, etc.) .

En este caso, el problema es el posterior procesamiento de este tipo de información especílica. Hemos visto que en 
la mayor parte de la comunicación no literaria, la información no se procesa conscientemente y se almacena así en la memoria, porque raramente se necesitará posteriormente en los procesos de recuperación. Además, no hay manera de conectar estructuralmente este tipo de estructuras superficiales con otra información del texto o de nuestros conocimientos. Por lo tanto, la tendencia será de que nuestra memoria y recursos de procesamiento sólo pueden almacenar y recuperar de manera muy restringida estos tipos de información estructural superficial, aun si las convenciones comunicativas requieren una atención especifica sobre tales estructuras. Así pues, ningún lector normal de literatura podrá reproducir, después de la lectura, palabra por palabra, un texto literario más o menos largo. Ni siquiera podrá reconocer la mayor parte de las oraciones usadas en el texto, aunque localmente les haya prestado mucha atención. Sólo ocasionalmente, como en un detalle estructural sobresaliente, podrá recordarse o reconocerse la selección de ciertas palabras o una estructura sintáctica. Desde luego, esta limitación del recuerdo puede disminuirse mediante procesos de repetición. Si leemos un discurso (literario o de otro tipo) varias veces, puede extenderse nuestra memoria de la información estructural (superficial), por lo menos durante cortos lapsos. Este tipo de capacidad realzada para las estructuras de superficie depende del destacamiento estructural particular de ciertas oraciones o secuencias: si las consideramos únicas, bellas, etc., se les puede asignar valores específicos en la memoria - debido al conjunto valorativo de nuestro conjunto cognoscitivo-, que significan más relaciones estructurales para aquellas oraciones; y por lo tanto una mayor recuperabiliclad.

Esta explicación de la posibilidad de procesar tipos específicos de información a partir de textos, también explica por qué la mayoría de poemas son relativamente cortos, un asunto muy poco tratado cuando se habla de la poesía. Cuando necesitamos más recursos de la memoria para procesar y almacenar información estructural (superficial), sólo es posible que funcione la memoria para esas palabras, frases y oraciones específicas $\rightarrow y$ las diversas operaciones fonoló. 
gicas, grafémicas y sintácticas en que se basan- cuando la cantidad de información es relativamente baja. Y por la misma razón, las convenciones literarias requieren que los poemas se lean con mayor atención, y más repetidamente (aprendidos de memoria), que por ejemplo las novelas. En poemas más largos o en novelas en general esperaremos o necesitaremos otras formas de organización, por ejemplo un esquema narrativo, y desde luego, temas y asuntos en un nivel más global. Es, pues, típico de muchas clases de poesía que el procesamiento predominantemente local permita un grado más bajo de organización en el nivel global. Aunque podemos tal vez asignarle un fragmento de un tema global (un "concepto temático", tal como "vida", "muerte", "amor", "miseria", etc.), no siempre es posible resumir normalmente un poema de la misma manera que podemos resumir el significado global de una novela en su "trama". Esto quiere decir que un poema bien puede carecer de una macroestructura, dado que su procesamiento es (i) a corto plazo, (ii) estructural superficial, y por último (iii) localmente semántico en general.

Este último punto nos lleva a las características semánticas específicas del procesamiento del discurso literario. Aparentemente, algunos textos literarios no tienen una macroestructura semántica, o si acaso sólo un tipo muy fragmentario de macroestructura. En ciertos tipos de poesía esta "carencia" de coherencia global se equilibra con un procesamiento semántico específico en e' nivel local. Por ejemplo, puede darse el caso de que (i) la estructura de superficie no pueda traducirse fácilmente 0 sin ambigüedad a proposiciones, y (ii) que estas proposiciones derivadas de las respectivas (semi) oraciones no puedan fácilmente conectarse mediante relaciones condicionales específicas (incluyendo, por ejemplo, referentes idénticos), o mediante información proposicional de la memoria. Esto sólo es posible cuando el lector tiene tiempo, recursos y atención suficientes para "descodificar" las oraciones y las conexiones locales entre oraciones, por ejemplo dejando de lado la construcción del tema global del texto.

La primera tarea cognoscitiva, pues, de un lector de este 
tipo de poesía (o ciertos tipos de prosa), es llegar a una interpretación semántica de las oraciones del poema. Esto puede resuitar difícil debido a posibles semioraciones, violaciones de categorías, etc., y porque no hay redundancia ni información contextual, como suele suceder en la interpretación de semioraciones en la conversación cotidiana. De esta manera, la interacción usual entre estructuras sintácticas y semánticas en la comprensión puede bloquearse parcialmente, lo que puede llevar a una comprensión parcial.

En tal caso, el lector puede aplicar diversas estrategias. En primer lugar, puede dejar tal cual la interpretación parcial (la estrategia de "a ver qué pasa") y tratar de encontrar información en el resto del texto, que puede proporcionar las ligas que faltan. Esta información también puede darse en una macroestructura total o fragmentaria. La última posibilidad se aplicará especialmente cuando no pueden conectarse oraciones que han sido interpretadas de manera más o menos adecuada. En tal caso, escán ligadas sólo macroestructuralmente y no en lo local, por ejemplo mediante un concepto común. La segunda estrategia es la de considerar la interpretación parcial como una base para conjuntos de interpretación libre. Un conjunto de interpretación libre es un conjunto de significados posibles, cuyos significados parciales son partes constituyentes compatibles. La variación personal de interpretaciones de este tipo, desde luego, puede ser muy alta; pero, debido a los principios pragmáticos y socioculturales básicos, esta variación personal no es prohibicla, dado que no lleva a conflictos en la (inter) acción futura del lector. Conocemos este tipo de estrategia, que a veces es necesaria inciuso normativamente, bajo términos como "ambigüedad", "polivalencia", etc., en la interpretación literaria. Una tercera estrategia es más restrictiva y requiere que el lector, aun con la ayuda de la interpretación parcial, intente encontrar "el" significado de una oración o de una secuencia de oraciones. Esta tarea tiene un carácter muy semejante a lia de resolución de problemas, ya que no hay una interpretación completa, inmediata y obvia, a partir del texto (otras oraciones y macroestructuras) ni de: contexto (conocimiento del mundo, del autor o de otros 
textos del autor). La estrategia de la búsqueda semántica en este caso requiere la eliminación de varios significados del conjunto de interpretación libre, para lograr una coherencia posible del texto, ya sea local o global. Desde luego, no siempre se dará el caso de que un lector, por ejemplo al explorar conjuntos de asociaciones, llegue al supuesto significado intencional de una oración, pero por lo menos podrá elaborar una hipótesis acerca de la interpretación que queda mejor. Algo parecido sucede en la interpretación de expresiones metafóricas de un texto, un problema cognoscitivo específico que no podemos tratar aquí.

Aunque se ha dicho antes que la interpretación cognoscitiva de ciertos tipos de poesía es predominantemente local, las distintas posibilidades de interpretación parcial en este nivel local pueden requerir que el poema sea también, o aun exclusivamente, interpretable en el nivel global. Fs decir que, aunque las oraciones relacionadas entre sí no tengan mucho sentido (coherencia), en conjunto claramente expresen o impliquen una o más macroestructuras (fragmentarias) en común, o sea un "tema" en el sentido tradicional de ese término.

La interpretación cognoscitiva de la prosa narrativa tiene características bastante diferentes. Con la excepción de algunos tipos de prosa moderna, la interpretación de oraciones y las conexiones entre ellas pocas veces será parcial: en el nivel local sabemos en general de qué se trata el relato. Sin embargo, los principios comunicativos generales del discurso literario (ritual) presentados más arriba permiten una atención (modestamente) destacada para las estructuras de superficie y estructuras locales en general, tales como variaciones estilisticas especificas (selección de palabras, complejidad de las oraciones, conexiones entre oraciones, etc.) . En lo que se refiere a la lectura de poesía, estos procesos pueden resultar en valoraciones locales (que posiblemente determinen el "placer" local) del texto, que en parte pueden ser las convenciones literarias normales. Con frecuencia 
se dirá que precisamente este procesamiento a nivel local es la diferencia específica entre relatos literarios (rituales) y no literarios (naturales), pero esto necesitaría una verificación empírica: bien puede ser que nuestro "placer" de escuchar relatos cotidianos se determine también por su "estilo" local.

La complejidad de la narrativa (Iiteraria), sin embargo, requiere que el procesamiento senántico también se dé en el nivel global: la formación de macroestructuras es necesaria, porque sin ellas no se sabría de qué se trata el relato. En principio funcionan las mismas macrorreglas de los otros tipos de discursos. Pero, una vez más en este macronivel, la interpretación puede ser parcial. Puede ocurrir que las distintas oraciones (proposiciones) definan, no sin ambigüedad, una macroproposición específica o un conjunto de ellas: el lector no sabrá exactamente de qué trata el relato, o Ie asignará varias interpretaciones globales (libres). Pero para comprender el resto deI texto, es necesario que se construya por lo menos una posible macroestructura hipotética; si no, el resultado puede ser una absoluta incoherencia cognoscitiva, por lo menos en el nivel global. El lector, entonces, estará obligado a hacer sólo una interpretación local, - a aceptar una interpretación global parcial, como por ejemplo un "tema" o "ambiente" o descripción global de un agente del discurso.

Por último, puede darse el caso en este nivel de interpretación de que la lógica condicional del texto sea perturbada: ciertas proposiciones o secuencias pueden haber sido expresadas en un orden transformado, es decir sin las indicaciones usuales de retrospección, anticipación etc. En este caso el lector también tiene una tarea específica en ija resolución del problema, a saber la reordenación semántica, de acuerdo con las ligas condicionales más probables entre (macro) proposiciones. Esto es necesario porque la interpretación de la coherencia de un texto está determinada por una (re) construcción conceptual de las secuencias de los hechos y las acciones representadas. Por lo tanto, el lector debe transformar el texto en algún tipo de ordenación semántica normal. En la narrativa, este tipo de transformaciones semánticas 
y sus interpretaciones cognoscitivas en general vienen acompañadas por (super)estructuras esquematicas transformadas, como por ejemplo una resolución al principio del texto $\mathrm{y}$ el ambiente y la complicación después. También aquí el lector tenderá a restaurar el orden esquemático canónico, en la forma en que está almacenado en su conocimiento general del discurso en la memoria. Estos tipos de transformaciones semánticas y esquemáticas implican no sólo la reordenación (permutación), sino también supresiones de distintos tipos, como sucede en el nivel de las oraciones. Una novela puede no tener un "final" (esperado), lo que es una categoría normal de "valoración" o "coda". Las mismas categorías semánticas descritas anteriormente se aplican en este caso: el lector buscará una (macro) proposición posible que no aparece, o aceptará un conjunto de interpretación libre.

Estos distintos tipos de procesos semánticos, esquemáticos y estructurales de superficie que funcionan en la interpretación de textos literarios tienen también consécuencias específicas en la memoria y el reciuerdo. Hemos ya indicado que la memoria es limitada para las estructuras de superficie y locales (estilísticas, etc.). La paradoja de este hecho es que nuestra memoria para el discurso literario ante todo afectará aquellas características que en general se consideran de primera importancia en la comunicación literaria: tenderemos a recordax de una novela la trama global, y no (cada una de) las manifestaciones estructurales superficiales estilísticas de un relato en el nivel local. Cuando mucho, tendremos un recuerdo global de los rasgos estilísticos más importantes, o el recuerdo incidental de un detalle sobresaliente. Podemos decir, pues, que en el procesamiento literario, más que en otros tipos de comunicación, la comprensión, el almacenamiento, la repetición y el recuerdo (y la reproducción: contarlo) cognoscitivos se orientan hacia la asignación de relevancia a los detalles sobresalientes. Esto sólo es posible mediante otros recursos (atención), ensayo/repetición (aun en el texto) y la liga estructural de ese detalle con la experiencia personal de sucesos o valoraciones semejantes. 


\section{Comentario final}

Se ha mostrado en la sección anterior que muchos rasgos conocidos de la comunicación literaria pueden formularse en términos de los procesos cognoscitivos que determinan la comprensión del discurso. También hemos visto que en la comprensión literaria debemos afrontar muchos rasgos interesantes, incluyendo la interpretación de estructuras incompletas, transformaciones semánticas, falta de coherencia local $y / 0$ global y transformaciones esquemáticas.

Queda claro que eśta presentación ha sido muy informal e incompleta: apenas hemos rozado la superficie (y esto no es sólo una fórmula perteneciente a la categoría "conclusión" de una ponencia). La investigación en el área de comprensión del discurso está en sus comienzos, y la investigación paralela sobre la comprensión del discurso literario virtualmente no existe: no hay modelos téóricos y sólo muy pocos experimentós tentativos sobre la comprensión real del discurso literario. Nuestro objetivo no fue sino el de indicar brevemente cuáles son los procesos generales de información cognoscitiva, que también, en principio, desempeñan una función en la comprensión de los textos literarios; al mismo tiempo quisimos sugerir en dónde podrían encontrarse otros procesos. Este último comentario no implica que procesos semejantes no desempeñen una función en la comprensión de otros tipos de discursos: las operaciones retóricas y de estilo en todos los niveles ocurren en cualquier uso de la lengua. Sólo significa que nuestro modelo general de procesamiento del discurso debería refinarse para manejar el análisis, la comprensión y el almacenamiento en la memoria de cales estructuras especificas. Lo mismo puede decirse respecto de los distintos efeclos de estos procesos sobre opiniones, raloraciones y actitudes.

Teun A. Yan DIJK 


\section{BIBLIOGRAFIA}

Para mayores referencias acerca de las distintas acciones, principios y modelos cognoscitivos de psicología usados en esta ponencia, el lector puede consultar las siguientes obras:

WALTER KNorsca, The Representation of Meaning in Memory. (Hillsdale, N. J.: Erlbaum, 1974).

Bonne Meyer, The Organization of Prose and its Effects on Memory (Amsterdam: North Holland, 1975).

Marcel Just \& Patricia Carpenter, eds., Cognitive Processes in Comprehension (Hillsdale, N. J.: Erlbaum, 1977) (Norwood, N. J.: Ablex, 1977).

Revista Discourse Processes y la serie de libros editados por Roy $O$. Freedle (Norwood N. J.: Ablex).

Para mi propio trabajo en esta área (en parte en colaboración con Walter Kintsch), ver por ejemplo:

Teun A. van Dijk \& Walter Kintsci, "Cognitive Psychology and Discourse", en W. U. Dressler, ed., Current Trends in Texilin. guistics (Berlín/Nueva York; De Gruyter, 1977), pp. 61-80.

WaLter Kintsch \& Teun A. VAN Dijk, "Towards a Model of Discourse Comprenhension and Production", Psychological Review 85 (1978) 363-394.

TEUN A, VAN DiJK, "Semantic Macro-Structures and Knowledge Frames in Discourse Comprehension". In: Just \& Carpenter, eds., op. cit., pp. 3.32.

Teun A, van Dijk, Macrostructures (Fillsdale, N, J: Erlbaum, 1980).

Aunque todos estos trabajos tratan, en especial, de los aspectos específicos de la producción y la comprensión del discurso, debe recordarse que presuponen los estucios psicológicos y psicolingüísticos sobre la producción y la comprensión del lenguaje, por ejemplo de las oraciones. Para un panorama reciente de este trabajo; el lector podría consultar:

Herbert H. Glark \& Eve Clark, Psychology and Language (Nueva York: Harcourt Brace \& Co., 1977).

Dada la importancia del conocimiento (marcos, etc-) para la comprensión del discurso, también debería consultarse:

Daniel G. Brobow \& Allan Collins, eds., Representation and Understanding (Nueva York: Academic Press, 1975).

Roger Schank \& Robert Abrlson, Scripts, Plans, Goals and Understanding (Hillsdale, N. J.: Eribaum, 1977). 
Para antecedentes generales de la lingüistica del texto, cf. mi libro: Text and Context (Londres: Longmans, 1977). En español: Texto y contexto, Madrid: Cátedra, 1980. 